\title{
Relativistic microburst storm characteristics: Combined satellite and ground-based observations
}

\author{
Sarah Dietrich, ${ }^{1}$ Craig J. Rodger, ${ }^{1}$ Mark A. Clilverd, ${ }^{2}$ Jacob Bortnik, ${ }^{3}$ and Tero Raita ${ }^{4}$ \\ Received 3 June 2010; revised 20 September 2010; accepted 28 September 2010; published 17 December 2010.
}

[1] We report a comparison of Solar Anomalous Magnetospheric Particle Explorer

detected relativistic electron microbursts and short-lived subionospheric VLF

perturbations termed FAST events, observed at Sodankylä Geophysical Observatory, Finland, during 2005. We show that only strong geomagnetic disturbances can produce FAST events, which is consistent with the strong link between storms and relativistic microbursts. Further, the observed FAST event perturbation decay times were consistent with ionospheric recovery from bursts of relativistic electron precipitation. However, the one-to-one correlation in time between microbursts and FAST events was found to be very low $(\sim 1 \%)$. We interpret this as confirmation that microbursts have small ionospheric footprints and estimate the individual precipitation events to be $<4 \mathrm{~km}$ radius. In contrast, our study strongly suggests that the region over which microbursts occur during storm event periods can be at least $\sim 90^{\circ}$ in longitude ( $\sim 6 \mathrm{~h}$ in magnetic local time). This confirms earlier estimates of microburst storm size, suggesting that microbursts could be a significant loss mechanism for radiation belt relativistic electrons during geomagnetic storms. Although microbursts are observed at a much higher rate than FAST events, the ground-based FAST event data can provide additional insight into the conditions required for microburst generation and the time variation of relativistic precipitation.

Citation: Dietrich, S., C. J. Rodger, M. A. Clilverd, J. Bortnik, and T. Raita (2010), Relativistic microburst storm characteristics: Combined satellite and ground-based observations, J. Geophys. Res., 115, A12240, doi:10.1029/2010JA015777.

\section{Introduction}

[2] The dynamics of Earth's Van Allen radiation belts are governed by a number of competing acceleration and loss mechanisms. Particle fluctuation often coincides with a period of disturbance caused by a geomagnetic storm, with the outer belt flux frequently decreasing during storm onset, followed by a gradual repopulation during the recovery period [Baker et al., 1986; Li et al., 1997]. However, this is not always the case; Reeves et al. [2003] found that the poststorm outer belt relativistic electron flux levels can increase (seen to occur for $53 \%$ of events studied), decrease $(19 \%)$ or have no significant change $(28 \%)$, relative to prestorm levels. The flux of the outer belt relativistic electron population at geostationary orbits, defined as having energies $>1 \mathrm{MeV}$, is seen to drop over a period of hours during geomagnetic storms [Onsager et al., 2002]. Multiple mechanisms may contribute to these decreases and it has

\footnotetext{
${ }^{1}$ Department of Physics, University of Otago, Dunedin, New Zealand. ${ }^{2}$ Physical Sciences Division, British Antarctic Survey, NERC, Cambridge, UK.

${ }^{3}$ Department of Atmospheric and Oceanic Sciences, University of California, Los Angeles, California, USA.

${ }^{4}$ Sodankylä Geophysical Observatory, University of Oulu, Sodankylä, Finland.

Copyright 2010 by the American Geophysical Union. 0148-0227/10/2010JA015777
}

been shown that losses to the atmosphere are likely to be a contributing factor [Green et al., 2004; Clilverd et al., 2006].

[3] Numerical modeling predicts Relativistic Electron Precipitation (REP) to penetrate into the atmosphere to altitudes of 40-60 km, lower altitudes than most other magnetospheric particles are able to reach [Baker et al., 1987; Callis et al., 1991], depositing large enough amounts of energy so as to dominate all other ionization sources at this altitude range. REP events can occur across a wide range of time scales, lasting from minutes to hours, or taking the form of a brief microburst $(<1 \mathrm{~s})$ of precipitating electrons. The occurrence of relativistic microbursts has been studied since they were first reported by Brown and Stone [1972], but there are still significant unknowns. The first reports of relativistic microbursts (as distinct from those in the tens of $\mathrm{keV}$ range) appear to have been made by the S81-1 satellite [Imhof et al., 1992]. Observations from the Solar Anomalous Magnetospheric Particle Explorer (SAMPEX) have helped to determine some characteristics of REP microbursts, showing that they are spatially small [Blake et al., 1996]. Nakamura et al. [2000] found that microbursts tend to occur during storm time in the local dawn sector and are likely to be produced through interactions with electron whistler mode waves. Microburst production has also been linked to interaction with chorus waves [Lorentzen et al., 2001a; O'Brien et al., 2004; Bortnik and Thorne, 2007], which occur predomi- 


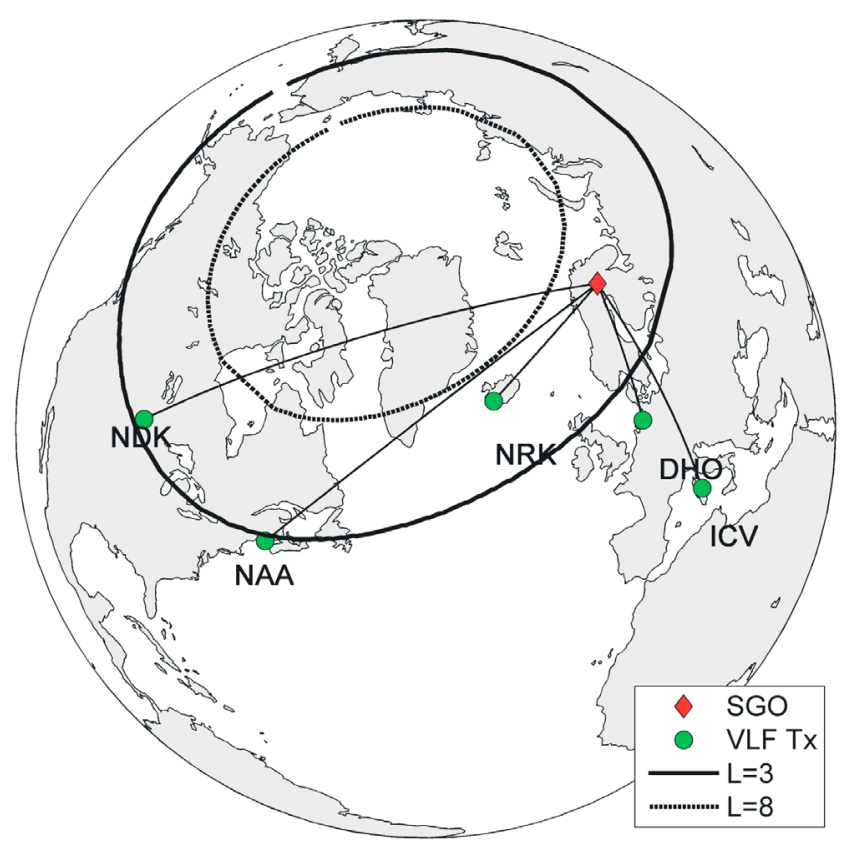

Figure 1. Map of the transmission paths of the five VLF signals that were used in this study. During the December 2004 to July 2005 period the AARDDVARK SGO receiver, indicated by a red diamond, was recording signals from the five transmitters that are marked by green circles. The $L=$ 3-8 range across which SAMPEX typically detects REP activity is enclosed between the two ellipses.

nantly in the dawn sector. The majority of geomagnetic storms show a sharp increase in microburst activity, tending to occur at lower $L$ during the storm onset, then slowly moving outward during the recovery period [Johnston and Anderson, 2010; Nakamura et al., 2000]. At this stage it is unclear from spacecraft data how large a spatial region is affected when relativistic microbursts take place. However, estimates have shown that relativistic microbursts could totally deplete the relativistic electron population of the outer belt during a geomagnetic storm [Lorentzen et al., $2001 \mathrm{~b}$; O'Brien et al., 2004]. Further information is required to understand the nature of magnetospheric relativistic electron losses [Thorne et al., 2005], requiring additional (and preferably) simultaneous measurements of microburst characteristics.

[4] As there are many suggestions but little certainty about the behavior of microbursts, methods of sensing REP at its lowest penetration altitude would be advantageous. Ionization levels at the $40-60 \mathrm{~km}$ altitude range can be difficult to probe, with one of the few effective monitoring techniques being the use of long-range Very Low Frequency (VLF) signals. VLF waves propagate by reflecting between the Earth's surface and the lower edge of the ionosphere, traveling in what is effectively an Earth-ionosphere waveguide. The altitude of the lower boundary of the ionosphere varies with solar zenith angle and local geomagnetic conditions, having an approximate value of $70-85 \mathrm{~km}$. Hence, REP tends to penetrate to below the lower ionospheric boundary, causing a pronounced increase in ionospheric ionization in the region of the precipitation event. Any VLF signal whose propagation path passes through the ionization change region will be perturbed in amplitude and phase, and as a result imprinted with an indication of REP activity. Recent studies of subionospheric VLF signals have found perturbation signatures of $\sim 1 \mathrm{~s}$ to occur during periods of geomagnetic disturbance [Clilverd et al., 2006; Rodger et al., 2007]. These perturbations have been termed FAST events and are thought to be the first documented ground-based detection of REP microbursts. It is hypothesized that each FAST event is the signature of one microburst, with a "rainstorm" of multiple microburst "raindrops" occurring in the area local to the receiver.

[5] In this paper we make a comparison between the characteristics of FAST events detected by subionospheric VLF and microbursts detected on a satellite, to determine the validity of the hypothesis that FAST events are the subionospheric signature of relativistic electron microburst precipitation events. Correlations in space and time will be examined to determine as much as possible about the nature of FAST events and to expand upon what is currently known about microbursts. In particular, the potential size of a single REP microburst, or "raindrop" size, is investigated, as is the size of the region across which microbursts can occur simultaneously, the "rainstorm" size.

\section{Instrumentation}

[6] This study combines subionospheric VLF signals and satellite data recorded from December 2004 to June 2005. We examine subionospheric VLF data detected at a VLF receiver located at the Sodankylä Geophysical Observatory (SGO), in Finland $\left(67.4^{\circ} \mathrm{N}, 26.4^{\circ} \mathrm{E}, L=5.3\right)$. We use signals from transmitters located in Europe and North America, i.e., NDK $\left(46.4^{\circ} \mathrm{N}, 98.3^{\circ} \mathrm{E}, L=3.3\right.$; North Dakota, USA; $\left.25.2 \mathrm{kHz}\right)$, NAA $\left(44.6^{\circ} \mathrm{N}, 67.3^{\circ} \mathrm{E}, L=2.9\right.$; Maine, USA; $\left.24.0 \mathrm{kHz}\right)$, NRK $\left(64.2^{\circ} \mathrm{N}, 21.9^{\circ} \mathrm{W}, L=5.6\right.$; Keflavik, Iceland; $\left.37.5 \mathrm{kHz}\right)$, DHO $\left(53.1^{\circ} \mathrm{N}, 7.6^{\circ} \mathrm{W}, L=2.4\right.$; Ramsloh, Germany; $23.4 \mathrm{kHz})$ and $\mathrm{ICV}\left(40.9^{\circ} \mathrm{N}, 9.8^{\circ} \mathrm{W}, L=1.5\right.$; Tavolara Island, Italy; $20.27 \mathrm{kHz}$ ). The transmitter locations and their signal transmission paths to SGO are shown in Figure 1. The SGO VLF receiver is part of the Antarctic-Arctic Radiation belt Dynamic Deposition VLF Atmospheric Research Konsortia (AARDDVARK) network. Further information about the AARDDVARK network is given by Clilverd et al. [2009] and is available the AARDDVARK website at http://www. physics.otago.ac.nz/space/AARDDVARK_homepage.htm.

[7] The Solar Anomalous and Magnetospheric Particle Explorer (SAMPEX) satellite carries the Heavy Ion Large Telescope (HILT), which gives high sensitivity and $30 \mathrm{~ms}$ time resolution measurements of the flux of $>1.05 \mathrm{MeV}$ electrons [Klecker et al., 1993]. While the Si-Li detectors are dominated by relativistic electrons during passes through the outer radiation belt, they may also be contaminated by protons during solar proton events. Monte Carlo calculations predict that $1 \mathrm{MeV}$ electrons see the HILT as having an effective geometric factor of $\sim 100 \mathrm{~cm}^{2}$ sr [Blake et al., 1996; Nakamura et al., 1998], a substantial viewing window that is ideal for studying energetic electron precipitation. HILT data with a high-rate sampling period of $20 \mathrm{~ms}$ was used in our investigation. The detectors on HILT saturate at a particle flux of $10^{4}$ electrons $\mathrm{cm}^{-2} \mathrm{~s}^{-1} \mathrm{sr}^{-1}$. 
Table 1. Properties of the Five FAST Periods Found From a Survey of SGO AARDDVARK Observations Across December 2004 to June $2005^{\mathrm{a}}$

\begin{tabular}{|c|c|c|c|c|c|c|}
\hline Date & Day/Night & Occurrence (UT) & SPE Flux (pfu) & Decay Time (s) & KP & Dst (nT) \\
\hline 19 Jan 2005 & Night & 0116-0614 & 190 & 1.04 & 6.7 & -75 \\
\hline 21 Jan 2005 & Night & $1712-1950$ & 374 & 0.8 & 8.0 & -99 \\
\hline 4-5 April 2005 & Night & $2050-0230$ & 0.5 & 1.2 & 7.0 & -79 \\
\hline 8 May 2005 & Day & $1255-1516$ & 0.1 & 0.84 & 8.3 & -109 \\
\hline 15 May 2005 & Day & 0236-0908 & 3790 & 0.63 & 8.3 & -262 \\
\hline
\end{tabular}

${ }^{\mathrm{a}} \mathrm{A}$ GOES pfu $=$ proton flux unit $=>10 \mathrm{MeV}$ protons $\mathrm{cm}^{-2} \mathrm{str}^{-1} \mathrm{~s}^{-1}$.

[8] The SAMPEX orbital period is $\sim 96 \mathrm{~min}$ and the magnetic local time of the satellite repeats approximately every 80 days [Blake et al., 1996]. The SAMPEX satellite's low-altitude, polar orbit means that it passes through Earth's radiation belts four times with each orbit, totaling approximately 60 passes a day, at $\sim 24$ min a pass. SAMPEX has an orbital inclination of $81.7^{\circ}$ [Nakamura et al., 1998].

[9] HILT mainly views the Bounce and Drift Loss Cones (BLC and DLC, respectively), thus detecting electrons that will precipitate within at least one drift period (i.e., $\sim 15 \mathrm{~min}$ at $L=4$ for a $1.5 \mathrm{MeV}$ electron).

\section{FAST Events}

[10] Clilverd et al. [2006] presented what is thought to be the first ever ground based detection of relativistic electron microbursts. This study undertook an analysis of subionospheric VLF AARDDVARK data during a magnetospheric electron flux decrease that took place at 1710 UT on 21 January 2005. Short-lived VLF amplitude and phase perturbations observed during the flux decrease were termed FAST events. It has been reported that FAST events are consistent with the expected effects of microbursts of rela- tivistic electrons impacting on the atmosphere and scattering VLF transmissions, as shown by the modeling by Rodger et al. [2007]. Approximately 99\% of individual FAST events occurring during the 21 January 2005 storm were not coincident across different received signals [Clilverd et al., 2006]. The lack of event coincidence suggests that FAST events are the result of a precipitation "rainstorm" producing spatially small (tens of km or less) "raindrop"-like ionization density changes, caused by a physical process spanning a much larger region, i.e., many hundreds of kilometers in diameter [Clilverd et al., 2006], centered on or near Sodankylä.

[11] A search for FAST events across the time period December 2004 to June 2005 identified four additional periods in SGO AARDDVARK data. As with 21 January 2005, all four additional periods occurred during geomagnetic storms, with two occurring during the local day time. This is expected as the relativistic electron precipitation that is thought to cause FAST event signatures should penetrate so deeply into the ionosphere that they would be observable both during the local day and night. The characteristics of all five documented FAST periods are listed in Table 1 and an example of a single FAST event is shown in Figure 2.

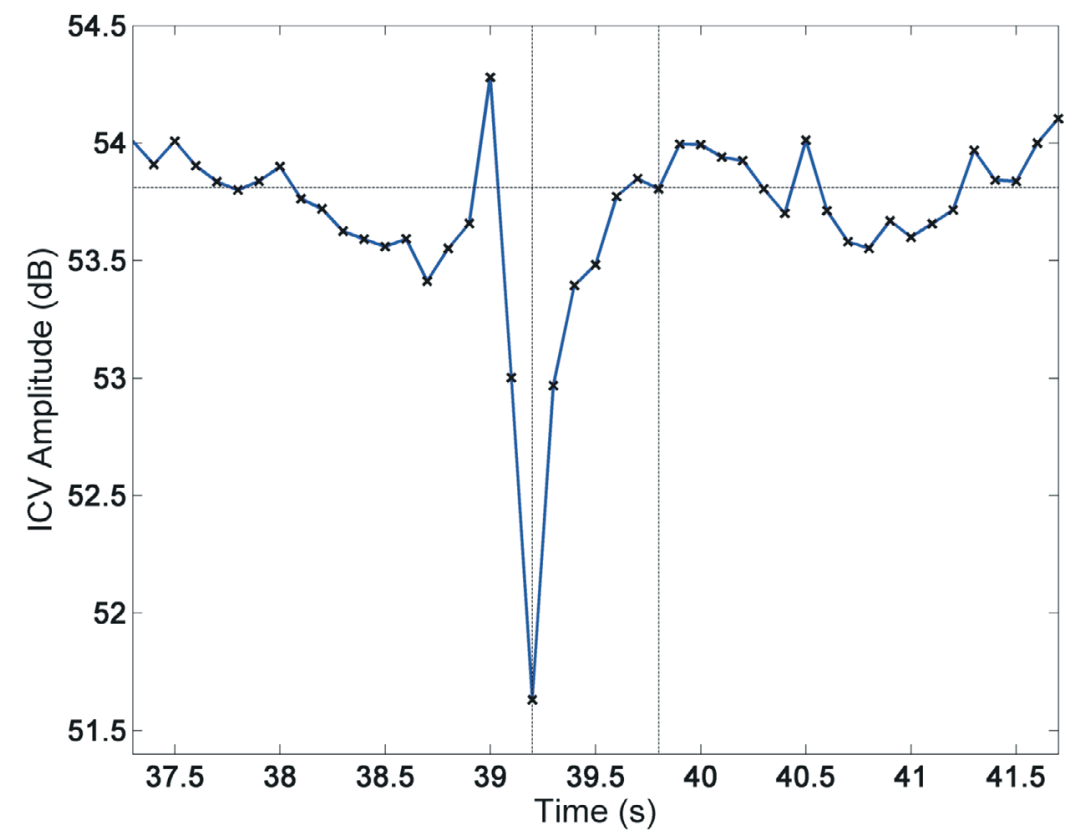

Figure 2. An example of a typical FAST event detected in SGO observations of ICV on 15 May 2005. Here the time is given in seconds from 0558:00 UT. The first dashed vertical line at $39.2 \mathrm{~s}$ indicates the start of the ionization decay, while the second vertical line at $39.8 \mathrm{~s}$ marks the perturbation's end, where the amplitude has returned to the background level. 
Included in Table 1 is the peak $>10 \mathrm{MeV}$ proton flux reported by the GOES for each period. No FAST event signatures were observed for $K_{P}<6$, suggesting that only strong geomagnetic disturbances can produce FAST events. This is consistent with the strong $D_{s t}$ link previously reported between storms and microbursts [O'Brien et al., 2003].

[12] The 21 January 2005 FAST events decayed over an average time period of $0.8 \mathrm{~s}$ each, while those for 4-5 April 2005 were observed to decay in an average of $1.2 \mathrm{~s}$ each. Rodger et al. [2007] showed the latter result is consistent with the modeled ionosphere recovery of ionization increases produced at altitudes as low as $40-60 \mathrm{~km}$ due to REP with energies $>2 \mathrm{MeV}$. The shorter 21 January 2005 decay time was explained by the upper parts of the REPproduced ionization changes being "masked" by excess ionization because of proton precipitation during a solar proton event. The time decays of the new FAST perturbations were examined, and contrasted with the geophysical conditions at the time.

[13] In Table 1 the shortest decay times are found for daytime ionospheric conditions when a solar proton event was occurring (15 May 2005), while the longest were for nighttime ionospheric conditions with essentially no precipitating proton flux (4-5 April 2005). A simple "cartoon model" would suggest that daytime REP produced ionization would have shorter decay times than that for nighttime REP. Similarly, REP produced ionization occurring during solar proton events would also have shorter decay times than non-SPE periods, due to increased high-altitude ionization levels perturbing the ionosphere. The FAST event decay times in Table 1 are consistent with the cartoon model of a rainstorm of REP microbursts.

\section{SAMPEX Microbursts}

[14] To investigate whether FAST events are caused by relativistic electron microbursts, a survey was undertaken of microbursts detected by the SAMPEX's HILT instrument during the FAST periods identified. The survey was confined to REP detected while HILT was viewing the bounce loss cone exclusively, as these microbursts represent local precipitation. This was defined by periods for which the mirror altitude of the SAMPEX-reported electron fluxes were less than $120 \mathrm{~km}$, indicating that the SAMPEX was only viewing the bounce loss cone.

[15] In the current study we use the $100 \mathrm{~km}$ altitude projection of SAMPEX's geomagnetic field line, as this provides the coordinates where any SAMPEX observed REP would precipitate into the atmosphere. As relativistic electron microbursts occur during large geomagnetic storms, some uncertainties might be expected in the field line mapping. We used the Definite/International Geomagnetic Reference Field (DGRF/IGRF), employing the GEOPACK software routines calculated for April 2005, to trace from the geomagnetic latitude and longitude of SAMPEX's location, down the magnetic field line to the top of the atmosphere. The effective top of the atmosphere was taken to be $\sim 100 \mathrm{~km}$, as in the SAMPEX data. To test the effect of a geomagnetic storm upon the $100 \mathrm{~km}$ altitude field line position, the $K_{P}$-dependent Tsyganenko magnetospheric field model was used [Tsyganenko, 1989]. The model was supplied with the maximum $K_{P}$ during the 4-5 April 2005
FAST period $\left(K_{P}=7\right)$. This showed that the maximum displacement was $<1 \mathrm{~km}$, thus the field line mapping to the atmosphere is sufficiently accurate for the purpose of this investigation.

[16] To determine whether a SAMPEX reported flux increase can be identified as a microburst, two criteria were employed as suggested by O'Brien et al. [2004]. It was required that HILT recorded a flux increase lasting $<1 \mathrm{~s}$ before decaying back to the level of the background flux, as well as measuring above a specified threshold flux during the increase. We follow O'Brien et al. [2004] and set the threshold flux increase, $J$, is $\sqrt{10}$ times the background population, $J_{0}$, that SAMPEX records, i.e.,

$$
J \geq \operatorname{sqrt}(10) \times J_{0}=3.16 J_{0}
$$

We also use O'Brien's method of establishing the baseline flux as the 10th percentile of the fluxes, while also placing a floor at a flux level of $10^{1} \mathrm{~cm}^{-2} \mathrm{str}^{-1} \mathrm{~s}^{-1}$ [O'Brien et al., 2004]. Any deviations from the J(DLC) level may represent bounce loss cone precipitation.

[17] We follow the approach outlined by Rodger et al. [2010] to examine the radiation belt electron populations observed by SAMPEX's HILT instrument. SAMPEX orbital data files include the IGRF-determined pitch angle at the spacecraft of a particle heading down the instrument boresight, which have been processed to produce a world map of median pitch angles with a $1^{\circ}$ latitude/longitude resolution. Using the IGRF model for an average SAMPEX altitude of $494 \mathrm{~km}$, we have created a similar world map for the angular width of the bounce and drift loss cones at the satellite. When these are combined with the HILT boresight pitch angle width of $68^{\circ}$ we can describe the geographical variation of the particle populations detected, taking into account the HILT viewing width.

[18] Figure 3 presents a world map of the changing radiation belt population observed by HILT. In Figure 3 "T" indicates trapped flux, "DLC" is drift loss cone, and "FL BLC" is field line bounce loss cone. Note that the FL BLC angle is defined as the largest of the two loss cone angles defined for the two hemispheres. Near the geomagnetic equator the instrument only measures fluxes inside the bounce loss cone (FL BLC), i.e., precipitating beneath the spacecraft, but for most of the globe it observes a mix of DLC and BLC populations [Klecker et al., 1993; Nakamura et al., 2000]. Note that above the SAMA the instrument detects part of the BLC, all of the DLC and a fraction of the trapped population. In contrast, in the Northern Hemisphere from about $85^{\circ} \mathrm{W}$ to $55^{\circ} \mathrm{E}$ the HILT detects only BLC fluxes, consistent with statements by Li et al. [1997]. While Figure 3 was made for the satellite mean altitude of $494 \mathrm{~km}$, very similar plots are produced for the full range of altitudes over which SAMPEX orbits $(\sim 450-530 \mathrm{~km})$.

[19] A survey of SAMPEX's HILT data found relativistic electron microbursts to occur during each of the FAST periods identified in the subionospheric data. These microbursts were sorted by their Northern Hemisphere mirror altitude to determine whether they were in the local drift or bounce loss cones, with the total number of microbursts detected and the number of those that were detected while viewing the BLC shown in Table 2. There is no simple relationship between the number of SGO FAST events and 


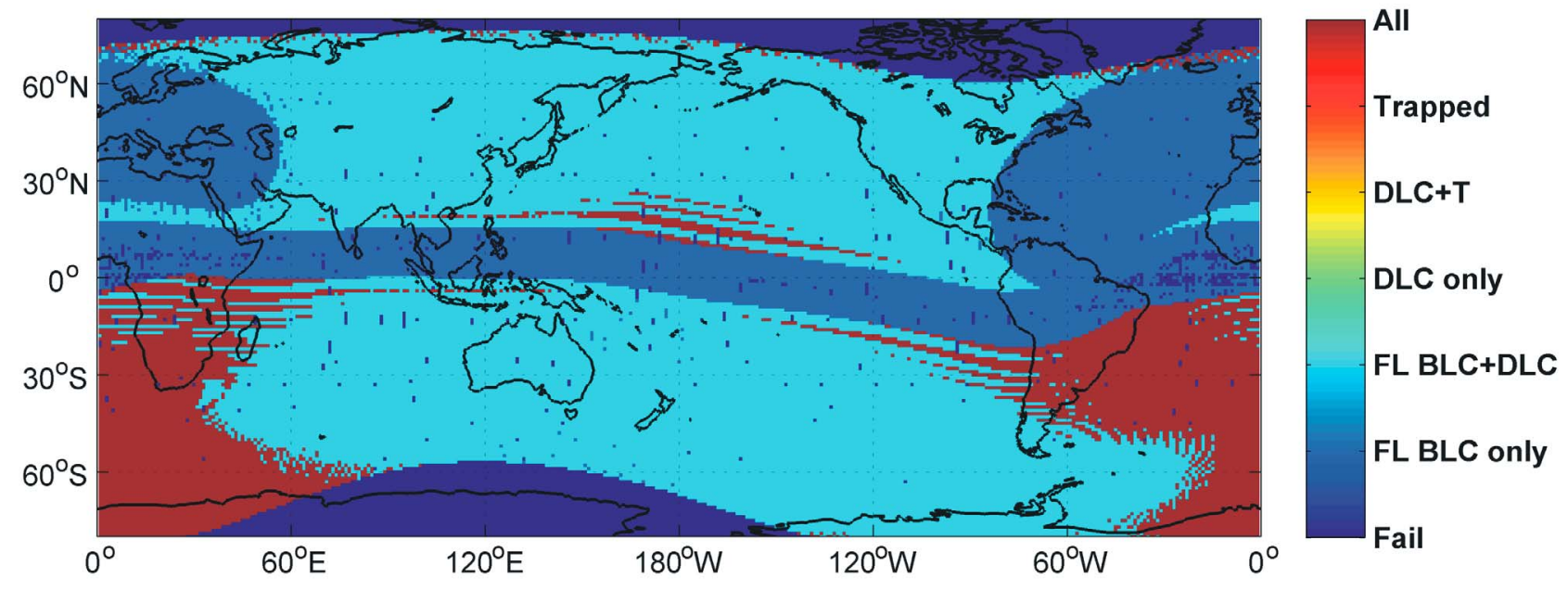

Figure 3. World map showing the changing radiation belt population observed by SAMPEX's HILT instrument. Here T indicates trapped flux, DLC is drift loss cone, and FL BLC is field line bounce loss cone. For most locations where there is a significant radiation belt flux, it observes a mix of DLC and FL BLC populations.

SAMPEX microbursts occurring on any given day. This is also true when considering $K_{P}$ and the number of microbursts, as is also seen with $K_{P}$ and the number of FAST events.

[20] The $L$ variation in the occurrence number and average flux of SAMPEX detected BLC microbursts in our data catalog is shown in Figure 4. Figure 4 (left) shows the recorded number of microbursts in each $L$ value range, which is in agreement with previously published results [Millan and Thorne, 2007, Figure 5b]. Figure 4 (right) shows the $L$ variation in the HILT reported BLC microburst intensities, in terms of the median increase in flux relative to the background. The dotted line shows the absolute flux increase with units of $\log _{10}\left(\right.$ electrons $\mathrm{cm}^{-2} \mathrm{~s}^{-1} \mathrm{str}^{-1}$ ), while the solid line shows the relative flux increase. A typical BLC microburst event observed in this study had a median $>1.05 \mathrm{MeV}$ flux of $\sim 400$ el. $\mathrm{cm}^{-2} \mathrm{~s}^{-1} \mathrm{str}^{-1}$, and was ten times larger than the background flux. Note that the mean flux increases are considerably larger, due to the very wide distribution of intensities. For example, the relative mean flux increase is 45 times above background for our BLC microburst events.

\section{Microburst Characteristics}

[21] To investigate the link between FAST events and REP microbursts, the BLC microbursts cataloged in this study were compared with the occurrence times of all SGO FAST events in our study period. For two events to be considered as coincident in time, the peak perturbations of the events must be separated by less than the average microburst duration, which we found to be $<0.29 \mathrm{~s}$. The percentage of temporal overlap of the two perturbations is not used as a criterion for determining coincident occurrence because the rise and fall of a FAST event appears to be determined by ionospheric conditions and can be partially masked by multiple FAST events occurring in a short time period. From a total of 219 BLC microbursts and 829 SGO FAST events, only two microbursts were found to be coincident with a FAST event, leading to a coincidence rate of $\sim 1 \%$.

[22] Periods of SAMPEX microbursts and SGO FAST events were also compared to look for event "patterns"; to test if there was a time offset in the SAMPEX data set and to see if any groups of events appeared to be coincident in time if the time axis was moved linearly. This effect was not seen. The drift loss cone microbursts cataloged were also tested to search for any one-to-one time correlations with FAST events, but none were found.

\subsection{Microburst Size}

[23] To determine the spatial extent of an individual microburst, two point measurements of the same microburst are needed. These two point measurements must be coincident in time and would ideally be separated by $\leq 10 \mathrm{~km}$, the previous SAMPEX-derived estimate for the horizontal extent of a single REP microburst [Blake et al., 1996]. If the SAMPEX size estimate is correct, two measurements that are coincident in time but separated by a greater distance will not detect the same microburst, but could give some insight into the maximum possible microburst size. Hence, points coincident in time but separated by a distance of up to $20 \mathrm{~km}$ will be considered in this study.

[24] In order to obtain two spatially close, temporally coincident point measurements for our study, SAMPEX must be reporting microbursts while passing over a monitored VLF transmission path, during a period in which

Table 2. SAMPEX Microburst Occurrence Numbers for the Five FAST Periods Occurring From December 2004 to June 2005, as Identified in This Study

\begin{tabular}{lcccc}
\hline 2005 & Kp & FAST Events & Microbursts & BLC Microbursts \\
\hline 19 Jan & 6.7 & 103 & 84 & 55 \\
21 Jan & 8.0 & 271 & 29 & 2 \\
4-5 Apr & 7.0 & 349 & 412 & 102 \\
8 May & 8.3 & 17 & 287 & 60 \\
15 May & 8.3 & 89 & 43 & 0 \\
\hline
\end{tabular}



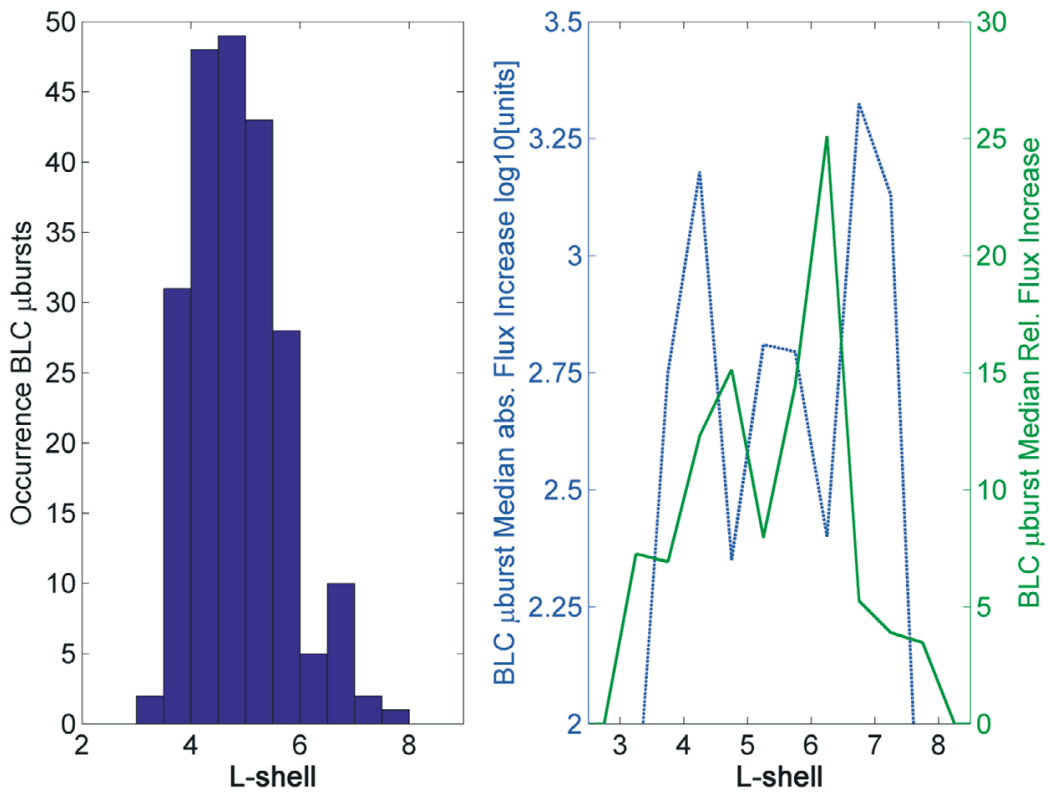

Figure 4. Occurrence of SAMPEX observed BLC microbursts examined in this study. (left) The variation in $L$ and (right) the median intensity of the microbursts observed. The dotted line shows the absolute flux increase with units of $\log _{10}\left(\right.$ electrons $\mathrm{cm}^{-2} \mathrm{~s}^{-1} \mathrm{str}^{-1}$ ), while the solid line shows the relative flux increase.

FAST events are observed. Of the five FAST periods identified in our study, only one SAMPEX pass was found to meet these conditions. At $\sim 0300$ UT on 5 April 2005, microbursts were detected while SAMPEX was viewing only the BLC on field lines which map to the atmosphere over the VLF transmitter-receiver path from ICV to SGO, as shown in Figure 5. The green crosses in Figure 5 are the field line traced positions of the 24 BLC microbursts that SAMPEX detected while it traveled northeast, crossing over the VLF transmission path (marked in black) and passing near the SGO receiver (marked by a red diamond). Thirteen of these 24 BLC microbursts were detected when SAMPEX was $\leq 20 \mathrm{~km}$ from the ICV path, hence these 13 fall within our criteria for "closeness." The two closest BLC microbursts occur at distances of $4.2 \mathrm{~km}$ and $4.3 \mathrm{~km}$ away from the path from ICV to SGO. However, no FAST events are observed at SGO coincident with these two microbursts, or for any of the other 11 of this set. The SGO observations of transmissions from ICV for this time period are shown in Figure 6 (top). Vertical thin lines mark the times of the first ten SAMPEX observed BLC microbursts for this time period. Clearly, no coincident FAST events are seen at these times. To provide context, Figure 6 (bottom) presents examples of FAST events on this transmitter signal, which occurred approximately $1 \mathrm{~h}$ earlier. The ten microbursts shown in Figure 6 (top) have a median flux of $\sim 3900 \mathrm{el} . \mathrm{cm}^{-2} \mathrm{~s}^{-1} \mathrm{str}^{-1}$, a value $\sim 10$ times greater than that found in section 4 . Even though the fluxes of these microbursts greatly exceed the typical flux we have identified, no coincident FAST events were seen. One interpretation of the lack of coincidence between BLC microbursts and FAST events in this case is that individual microbursts have radii $<4 \mathrm{~km}$; this estimate assumes that microbursts are roughly circular and each one is approximately the same spatial size. Our estimate is consis-

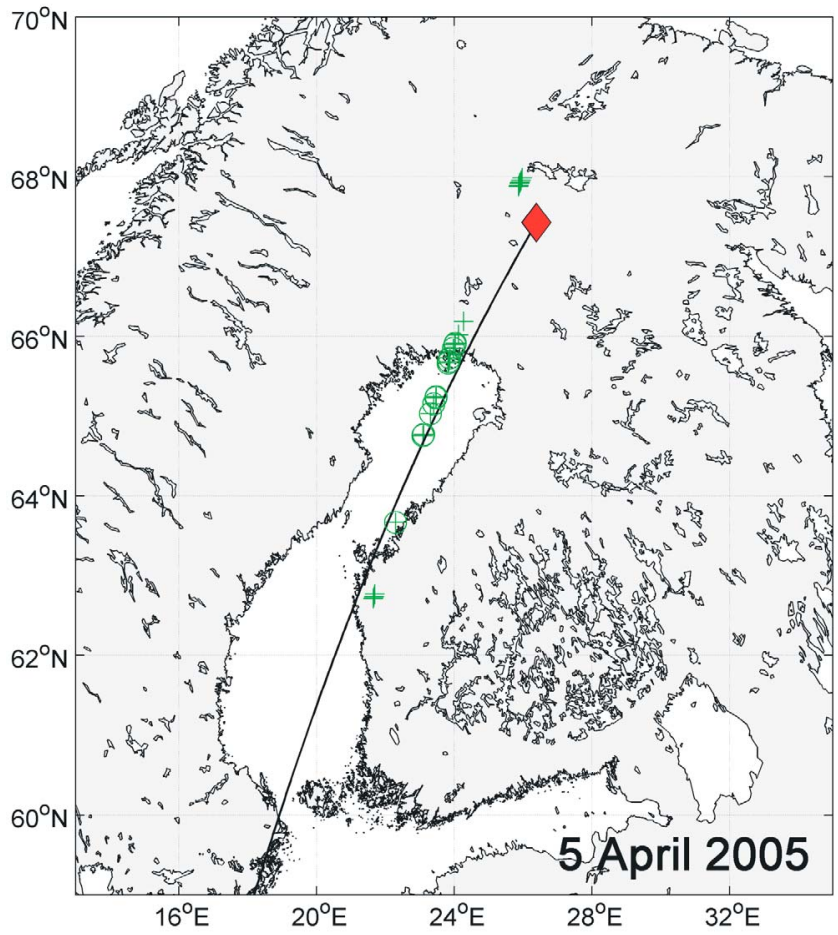

Figure 5. Map of the region around SGO in Finland (red diamond), showing a section of the ICV transmission path in black. The green crosses mark the positions of the SAMPEX satellite when it was detecting microbursts during 5 April 2005. The circled crosses are those within $20 \mathrm{~km}$ of the transmitter-receiver path. 

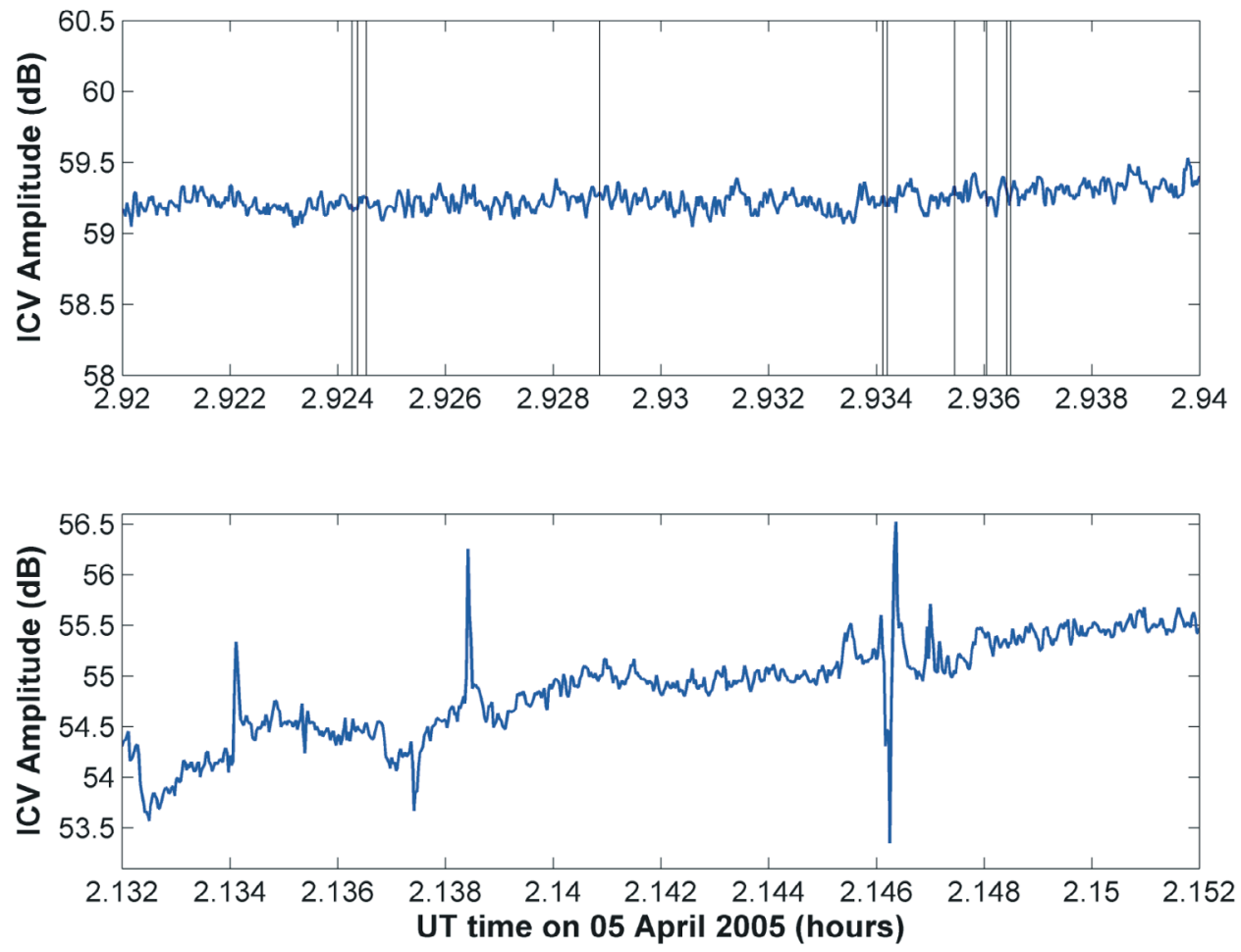

Figure 6. (top) The amplitude of the VLF signal from ICV received at SGO during the SAMPEX observations shown in Figure 5. Vertical lines indicate the occurrence of the ten BLC microbursts that were shown as the southernmost green crosses in Figure 5. (bottom) Examples of FAST events from the ICV channel.

tent with the Blake et al. [1996] suggestion of a diameter of $<10 \mathrm{~km}$, but at this point we have not been able to "catch a raindrop."

[25] During our analysis we also identified SAMPEX BLC microbursts that occurred on 19 January 2005, as SAMPEX was passing above western Iceland. The eastern part of Iceland hosts the U.S. Navy VLF transmitter NRK, which is monitored at SGO and at the AARDDVARK receiver in $\mathrm{Ny}$ Ålesund, Svalbard $\left(79^{\circ} \mathrm{N}, 11^{\circ} \mathrm{E}, L=18.3\right)$. While the NRK observations at SGO showed FAST events, those from Ny Ålesund did not, with no coincident BLC microbursts and SGO FAST events. This strongly suggests that the precipitation needs to occur close to the receiver to produce a detectable FAST event, as otherwise FAST events should have been seen in the data from both AARDDVARK stations. In this case SAMPEX reported BLC microbursts over Iceland and FAST events were reported by SGO. Given that these two points are roughly $50^{\circ}$ in longitude apart, it appears that the size of the rainstorm which produces microbursts may be very large. We consider this in more detail in section 5.2.

\subsection{Storm Size}

[26] If the storm size, the spatial extent of the magnetospheric process that is causing microbursts, is to be determined, a minimum of two point measurements are again needed. Detection of microbursts occurring at similar times while being spatially separated by $>10 \mathrm{~km}$, would help to determine the minimum size of a "microburst storm." Here we assume that FAST events are indeed relativistic electron microbursts, while we acknowledge that so far, we have been unable to "catch a raindrop." In this way we can attempt to measure the size of the storm driving microburst production.

[27] At about 1420 UT on 8 May 2005 SAMPEX moved into the North Atlantic region in which it views only the BLC. During this period, SGO had been reporting FAST events and, as expected, SAMPEX detected a series of relativistic BLC microbursts as it passed from about $L=4$ to $L=6$. The SAMPEX events occurred while the satellite was passing over the mouth of the Gulf of Saint Lawrence in eastern Canada. The simultaneous observation of REP precipitation at SAMPEX's location and SGO suggests either two widely separated storms or one single large storm spanning $94^{\circ}$ in longitude from the easternmost microburst position to $\mathrm{SGO}$, stretching across $5,061 \mathrm{~km}$ and spanning 2-12 magnetic local time (MLT). Further support for the existence of a single storm region comes from precipitation observed on 19 January 2005. During 0100-0600 UT SAMPEX detected BLC microbursts over the North East coast of North America and also in the North Atlantic. Across this time period SGO also observed FAST event activity. The field line traced locations of these microbursts are shown in Figure 7 as green circles, superimposed upon the orbital track for which SAMPEX is observing only the BLC (smaller blue circles). The position of SGO is shown by a red diamond. Here a single storm system of a minimum of $\sim 90^{\circ}$ in longitude again spanning a minimum of $\sim 6 \mathrm{MLT}$ seems most likely. Note that this is consistent with earlier 


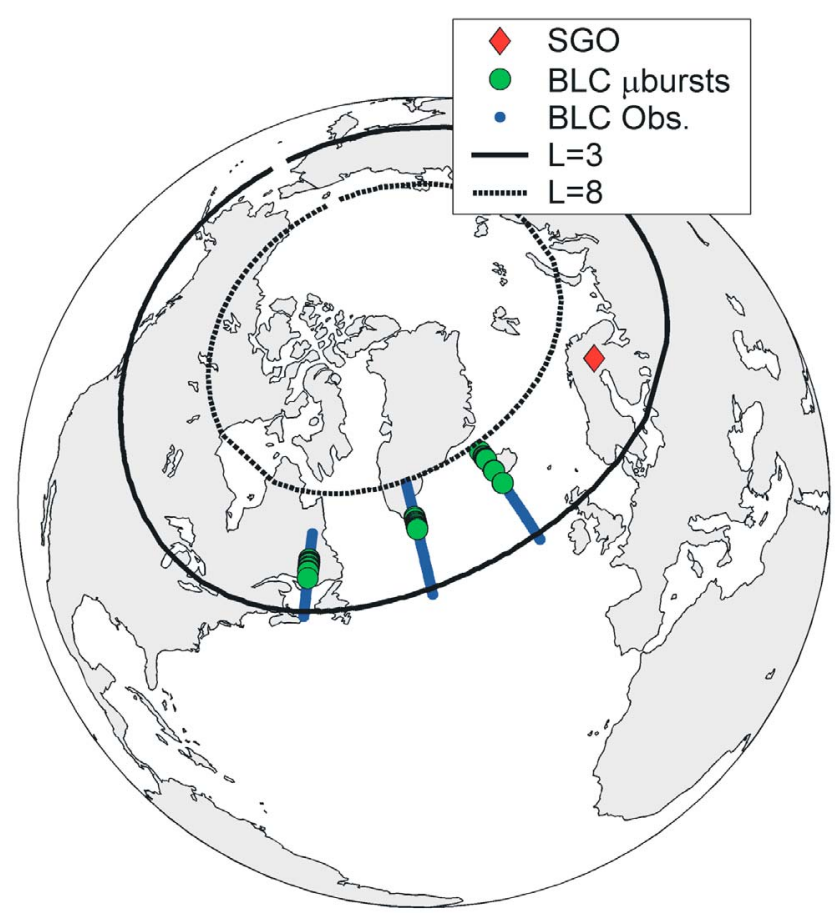

Figure 7. Locations of SAMPEX detected bounce loss cone microbursts (green circles) on 19 January 2005 which occurred in the time period FAST events were detected at SGO (red diamond).

SAMPEX studies which established that relativistic microbursts are most common from $\sim 6-12$ MLT [Millan and Thorne, 2007, Figure 5b], and suggests that such large storm sizes may well be typical. The MLT range and extent in which SAMPEX detects microbursts is quite similar to that reported for chorus whistler mode waves [Meredith et al., 2003], particularly for off-equatorial locations where wave-particle interactions with relativistic electrons are possible [Bortnik et al., 2007].

[28] We are currently unable to test if a storm can be any wider, due to the fixed receiver placement at SGO and due to the restricted longitude range in which SAMPEX detects only BLC microbursts (as shown in Figure 3). In addition, we do not currently have another receiver in the AARDDVARK network in the correct longitude and $L$ range, although a new deployment is expected in western Canada in October 2010 which should allow expanded microburst storm viewing.

\section{Discussion}

[29] Earlier work reported that FAST events detected by AARDDVARK subionospheric VLF have a one-to-one correlation of $\sim 1 \%-2 \%$ when observed across multiple VLF signals [Rodger et al., 2007]. This very low one-to-one correlation appears to support a small scale size of $\leq 4 \mathrm{~km}$ for an individual relativistic electron microburst and also suggests that the precipitation is occurring in a "rainstorm." There are some uncertainties concerning this interpretation, however. Previous studies have demonstrated how spatially small, highly conductive regions (produced by many order of magnitude increases in $\mathrm{D}$ region ionization levels) can produce high levels of scattering of subionospheric VLF transmissions [e.g., Rodger, 1999, 2003]. One example of this situation is the VLF perturbations produced by red sprites. In these cases, the ionization change can be located well off the transmitter-receiver great circle path and still lead to a significant VLF perturbation [Hardman et al., 1998]. Extreme cases of VLF perturbations caused by ionization changes occurring "behind" the receiver have been reported [Dowden et al., 1996]. As such, one might expect that the spatially small ionization changes produced by relativistic electron microbursts would lead to FAST events irrespective of whether they are very close to the transmitter-receiver great circle path or somewhere close to the receiver. In these cases, wide-angle scattering would cause a single ionization change located within a few $100 \mathrm{~km}$ of the receiver to produce coincident VLF perturbations on multiple transmitter paths. Clearly, however, this is not observed in our current study. One possible reason for this is that the expected maximum $\mathrm{D}$ region electron density change calculated for a reasonable representation of a typical relativistic electron microburst of $100 \mathrm{el} . \mathrm{cm}^{-2} \mathrm{~s}^{-1}$ $\operatorname{str}^{-1}$ [Rodger et al., 2007, Figure 5] is an increase of $\sim 20$ 40 times, while red sprites produce 4-6 order of magnitude electron density increases [e.g., Rodger and Nunn, 1999; Nunn and Rodger, 1999; Armstrong et al., 2000], in comparison with the ambient nighttime ionosphere. This suggests that a typical relativistic electron microburst is likely to be too small to lead to significant wide-angle VLF scattering.

[30] During each 24 min pass through the outer radiation belt, SAMPEX travels from $L=4$ to $L=6$, the region where the majority of microbursts occur, in just 1 min $45 \mathrm{~s}$. In contrast, the SGO receiver has a fixed position it can potentially detect FAST events at any time. This shows up the challenge in directly comparing ground-based and satellite observations of short-lived events occurring only during major geomagnetic storms. However, we can use the results shown in Table 2 to estimate an occurrence rate for FAST events and BLC microbursts, and thus compare the two data sets more closely. During the 5 event periods FAST events are typically observed at a rate of $\sim 0.6 \mathrm{~min}^{-1}$, while the BLC microbursts are observed at a typical rate of $\sim 8 \mathrm{~min}^{-1}$. These estimates suggest that only $\sim 8 \%$ of BLC microbursts are observable as FAST events in the subionospheric data. This might suggest that FAST event signatures are generated by the $\sim 10 \%$ of microbursts which have the largest precipitation flux, although the lack of any clear one-to-one linkage makes this suggestion quite speculative. In addition, based on the occurrence rates and event duration, one would expect FAST and BLC microbursts to agree in time by chance $\sim 4 \%$ of the time. Given that the observed rate of coincidence between FAST events and BLC microbursts determined earlier in this study was $\sim 1 \%$, it is likely that this coincidence rate is due to chance, rather than direct agreement. Thus it is not clear that we have the simultaneous observation of the same relativistic precipitation bursts from SAMPEX and SGO, even though both data sets indicate relativistic precipitation bursts are occurring during a given time window.

[31] Although the rate of occurrence of FAST events is low in comparison with BLC microbursts, the SGO data does provide some additional information regarding the time var- 


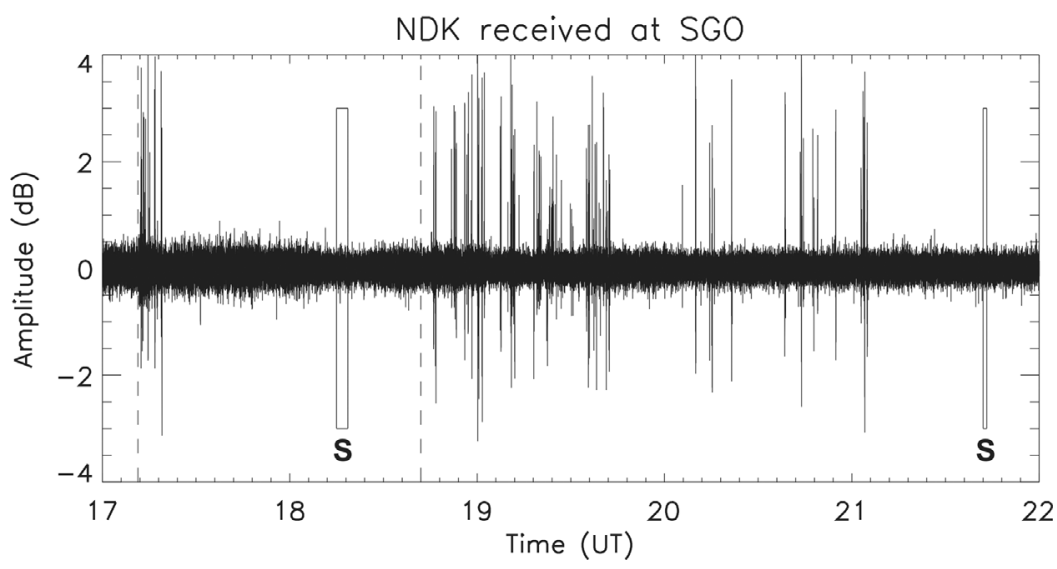

Figure 8. Observations of FAST events observed on transmissions from N. Dakota (NDK) at Sodankylä (SGO) on 21 January 2005. The two vertical dashed lines at 1712 UT and 1842 UT indicate the times of two solar wind pressure pulses. The two periods labeled "S" represent the times of SAMPEX observations in the BLC over the $L=3-8$ range.

iation of relativistic precipitation, which is difficult to determine from low-Earth orbit satellite data alone. Figure 8 is an example of the type of information that ground-based observations of relativistic precipitation detected through FAST events can provide. The plot shows FAST events on the transmitter NDK received at SGO from 1700 to 2200 UT on 21 January 2005. Vertical dashed lines indicate the times of large solar wind pressure pulses [e.g., Clilverd et al., 2007]. Two periods labeled "S" represent the times of SAMPEX observations in the BLC over the $L=3-8$ range. As can be seen from the plot, FAST events occur following the times of the pressure pulses, with only low levels of occurrence between the two shock events. During this storm period SAMPEX's orbit was such that it was unable to observe most of the time variation in the relativistic microburst activity that was occurring. Clearly, continuous ground-based observations can provide additional insight into the conditions required for microburst generation.

[32] There is currently a satellite mission in its planning stages, which aims to determine the spatial extent and energy dependence of relativistic and nonrelativistic electron microbursts. This project is the Focused Investigations of Relativistic Electron Burst Intensity, Range, and Dynamics (FIREBIRD) [Moretto, 2009] mission, to be launched in early 2012. A pair of satellites will be launched together, each carrying a solid-state detector with a large geometric factor measuring $30 \mathrm{keV}$ to $3 \mathrm{MeV}$ electrons. The two FIREBIRD satellites will gradually drift apart over the course of the mission, reaching a separation of $\sim 300 \mathrm{~km}$ [Moretto, 2009]. The FIREBIRD mission will provide a twopoint microburst detection system, and as such will be a further opportunity for two-point measurements of relativistic microbursts, following on from the recent ground based work. Combining the FIREBIRD observations with additional ground-based measurements may provide additional clarity to the storm size measurements.

\section{Conclusions and Summary}

[33] We have attempted to show that FAST events detected in subionospheric VLF observations are caused by relativistic electron microbursts, through a comparison with SAMPEX detected relativistic electron precipitation occurring during FAST event periods. We have also attempted to demonstrate the spatial extent of a single microburst raindrop and the size of an entire microburst storm.

[34] By building upon previous research into the nature of FAST events, it appears that FAST events are indeed caused by relativistic electron microburst precipitation. In this study we have shown that only strong geomagnetic disturbances can produce FAST events, consistent with the strong $D_{s t}$ link between storms and relativistic microbursts and we have shown that SAMPEX detects relativistic microbursts during the identified FAST event periods. In addition, the dependence of observed FAST event perturbation decay times on ionospheric conditions is also consistent with the subionospheric perturbations being caused by short-lived bursts of relativistic electron precipitation.

[35] However, this study suggests that only the strongest (i.e., highest flux) microbursts might produce an observable FAST event, such that there is little direct agreement between individual microbursts and FAST events. The one-to-one correlation in time between microbursts and AARDDVARK FAST events is very low $(\sim 1 \%)$, and occurs most likely by chance rather than a direct detection. In the one case where SAMPEX flew along a transmitter-receiver path, none of the BLC microbursts reported by SAMPEX corresponded in time to FAST events. One interpretation of this is that the individual microbursts have radii $<4 \mathrm{~km}$, which is consistent with earlier satellite-based findings. Our study strongly suggests that the magnetospheric process which generates relativistic microbursts is vastly larger than the individual bursts. Two examples are provided where the storm stretches $\sim 90^{\circ}$ in longitude and $\sim 6 \mathrm{~h}$ in MLT. This is particularly important given that Lorentzen et al. [2001b] used a $\sim 6$ MLT estimate of microburst storm size when showing that relativistic electron microbursts could flush out the entire radiation belt relativistic electron population in less than a day. Our findings support this previous work, which concluded that REP microbursts could be a highly significant loss mechanism for relativistic electrons during geomagnetic storms. 
[36] Acknowledgments. C.J.R. and M.A.C. would like to thank the International Space Science Institute (ISSI) of Bern, Switzerland, for providing the environment in which this paper could be completed. J.B. would like to thank T. P. O'Brien for his invaluable assistance in acquiring and reading the SAMPEX data and NSF for their kind support through grant ATM-0840178.

[37] Masaki Fujimoto thanks the reviewers for their assistance in evaluating this paper.

\section{References}

Armstrong, R. A., D. M. Suszcynsky, W. A. Lyons, and T. Nelson (2000), Multi-color photometric measurements of ionization and energies in sprites, Geophys. Res. Lett., 27, 653-656.

Baker, D. N., R. W. Klebesadel, P. R. Higbie, and J. B. Blake (1986), Highly relativistic electrons in the Earth's outer magnetosphere: 1. Lifetimes and temporal history 1979-1984, J. Geophys. Res., 91, 4265-4276.

Baker, D. N., J. B. Blake, J. D. Gorney, and P. R. Higbie (1987), Highly relativistic magnetospheric electrons: A role in coupling to the middle atmosphere?, Geophys. Res. Lett., 14, 1027-1030.

Blake, J. B., M. D. Looper, D. N. Baker, R. Nakamura, B. Klecker, and D. Hovestadt (1996), New high temporal and spatial resolution measurements by SAMPEX of the precipitation of relativistic electrons, Adv. Space Res., 18(8), 171-186.

Bortnik, J., and R. M. Thorne (2007), The dual role of ELF/VLF chorus waves in the acceleration and precipitation of radiation belt electrons, J. Atmos. Sol. Terr. Phys., 69, 378-368.

Bortnik, J., R. M. Thorne, and N. P. Meredith (2007), Modeling the propagation characteristics of chorus using CRRES suprathermal electron fluxes, J. Geophys. Res., 112, A08204, doi:10.1029/2006JA012237.

Brown, J., and E. Stone (1972), High-energy electron spikes at high latitudes, J. Geophys. Res., 77(19), 3384-3396.

Callis, L. B., D. N. Baker, J. B. Blake, J. D. Lambeth, R. E. Bouhner, M. Natarajan, R. W. Klebesadel, and D. J. Gorney (1991), Precipitating relativistic electrons: Their long term effect on stratospheric odd nitrogen levels, J. Geophys. Res., 96, 2939-2976.

Clilverd, M. A., C. J. Rodger, and T. Ulich (2006), The importance of atmospheric precipitation in storm-time relativistic electron flux drop outs, Geophys. Res. Lett., 33, L01102, doi:10.1029/2005GL024661.

Clilverd, M. A., C. J. Rodger, R. M. Millan, J. G. Sample, M. Kokorowski, M. P. McCarthy, T. Ulich, T. Raita, A. J. Kavanagh, and E. Spanswick (2007), Energetic particle precipitation into the middle atmosphere triggered by a coronal mass ejection, J. Geophys. Res., 112, A12206, doi:10.1029/2007JA012395.

Clilverd, M. A., et al. (2009), Remote sensing space weather events: The AARDDVARK network, Space Weather, 7, S04001, doi:10.1029/ 2008SW000412

Dowden, R. L., J. B. Brundell, C. J. Rodger, O. A. Molchanov, W. A Lyons, and T. Nelson (1996), The structure of red sprites determined by VLF scattering, IEEE Antennas Propag. Mag., 38(3), 7-15.

Green, J. C., T. G. Onsager, T. P. O’Brien, and D. N. Baker (2004), Testing loss mechanisms capable of rapidly depleting relativistic electron flux in the Earth's outer radiation belt, J. Geophys. Res., 109, A12211, doi:10.1029/2004JA010579.

Hardman, S. F., C. J. Rodger, R. L. Dowden, and J. B. Brundell (1998), Measurements of the VLF scatter pattern of the structured plasma of red sprites, IEEE Antennas Propag. Mag., 40(2), 29-38.

Imhof, W. L., H. D. Voss, J. Mobilia, D. W. Datlowe, E. E. Gaines, J. P. McGlennon, and U. S. Inan (1992), Relativistic electron microbursts, J. Geophys. Res., 97(A9), 13,829-13,837.

Johnston, W. R., and P. C. Anderson (2010), Storm time occurrence of relativistic electron microbursts in relation to the plasmapause, J. Geophys. Res., 115, A02205, doi:10.1029/2009JA014328.

Klecker, B., et al. (1993), HILT: A heavy ion large area proportional counter telescope for solar and anomalous cosmic rays, IEEE Trans. Geosci. Remote Sens., 31(3), 542-548, doi:10.1109/36.225520.

Li, X., D. N. Baker, M. Temerin, T. E. Cayton, E. G. D. Reeves, R. A. Christensen, J. B. Blake, M. D. Looper, R. Nakamura, and S. G. Kanekal (1997), Multisatellite observations of the outer zone electron variation during the November 3-4, 1993, magnetic storm, J. Geophys. Res., $102,14,123-14,140$.
Lorentzen, K. R., J. B. Blake, U. S. Inan, and J. Bortnik (2001a), Observations of relativistic electron microbursts in association with VLF chorus, J. Geophys. Res., 106, 6017-6027, doi:10.1029/2000JA003018.

Lorentzen, K. R., M. D. Looper, and J. B. Blake (2001b), Relativistic electron microbursts during the GEM storms, Geophys. Res. Lett., 28(13), 2573-2576.

Meredith, N. P., R. B. Horne, R. M. Thorne, and R. R. Anderson (2003), Favored regions for chorus-driven electron acceleration to relativistic energies in the Earth's outer radiation belt, Geophys. Res. Lett., 30(16), 1871, doi:10.1029/2003GL017698.

Millan, R. M., and R. M. Thorne (2007), Review of radiation belt relativistic electron losses, J. Atmos. Sol. Terr. Phys., 69, 362-377.

Moretto, T. (2009), Update on the NSF Cubesat program, CEDAR Post, 56, $3-4$.

Nakamura, R., K. Kamei, and Y. Kamide (1998), SAMPEX observations of storm-associated electron flux variations in the outer radiation belt, J. Geophys. Res., 103(A11), 26,261-26,269.

Nakamura, R., M. Isowa, Y. Kamide, D. N. Baker, J. B. Blake, and M. Looper (2000), SAMPEX observations of precipitation bursts in the outer radiation belt, J. Geophys. Res., 103(A7), 15,875-15,885.

Nunn, D., and C. J. Rodger (1999), Modeling the relaxation of red sprite plasma, Geophys. Res. Lett., 26, 3293-3296.

O'Brien, T. P., K. R. Lorentzen, I. R. Mann, N. P. Meredith, J. B. Blake, J. F. Fennell, M. D. Looper, D. K. Milling, and R. R. Anderson (2003), Energization of relativistic electrons in the presence of ULF power and $\mathrm{MeV}$ microbursts: Evidence for dual ULF and VLF acceleration, J. Geophys. Res., 108(A8), 1329, doi:10.1029/2002JA009784.

O'Brien, T. P., M. D. Looper, and J. B. Blake (2004), Quantification of relativistic electron microburst losses during the GEM storms, Geophys. Res. Lett., 31, L04802, doi:10.1029/2003GL018621.

Onsager, T. G., G. Rostoker, H.-J. Kim, G. D. Reeves, T. Obara, H. J. Singer, and C. Smithtro (2002), Radiation belt electron flux dropouts: Local time, radial, and particle-energy dependence, J. Geophys. Res., 107(A11), 1382, doi:10.1029/2001JA000187.

Reeves, G. D., K. L. McAdams, R. H. W. Friedel, and T. P. O’Brien (2003), Acceleration and loss of relativistic electrons during geomagnetic storms, Geophys. Res. Lett., 30(10), 1529, doi:10.1029/2002GL016513.

Rodger, C. J. (1999), Red sprites, upward lightning, and VLF perturbations, Rev. Geophys., 37(3), 317-336.

Rodger, C. J. (2003), Subionospheric VLF perturbations associated with lightning discharges, J. Atmos. Sol. Terr. Phys., 65(5), 591-606.

Rodger, C. J., and D. Nunn (1999), VLF scattering from red sprites: Application of numerical modeling, Radio Sci., 34, 923-932.

Rodger, C. J., M. A. Clilverd, D. Nunn, P. Verronen, J. Bortnik, and E. Turunen (2007), Storm time, short-lived bursts of relativistic electron precipitation detected by subionospheric radio wave propagation, J. Geophys. Res., 112, A07301, doi:10.1029/2007JA012347.

Rodger, C. J., M. A. Clilverd, J. Green, and M.-M. Lam (2010), Use of POES SEM-2 observations to examine radiation belt dynamics and energetic electron precipitation in to the atmosphere, J. Geophys. Res., 115, A04202, doi:10.1029/2008JA014023.

Thorne, R. M., T. P. O’Brien, Y. Y. Shprits, D. Summers, and R. B. Horne (2005), Timescale for MeV electron microburst loss during geomagnetic storms, J. Geophys. Res., 110, A09202, doi:10.1029/2004JA010882.

Tsyganenko, N. A. (1989), Determination of magnetospheric current system parameters and development of experimental geomagnetic models based on data from IMP and HEOS satellites, Planet. Space Sci., 37, $5-20$.

J. Bortnik, Department of Atmospheric and Oceanic Sciences, University of California, Los Angeles, CA 90095-1565, USA. (jbortnik@gmail.com) M. A. Clilverd, Physical Sciences Division, British Antarctic Survey, NERC, High Cross, Madingley Road, Cambridge CB3 0ET, England, UK. (macl@bas.ac.uk)

S. Dietrich and C. J. Rodger, Department of Physics, University of Otago, P.O. Box 56, Dunedin, New Zealand. (sdietrich@physics.otago.ac.nz crodger@physics.otago.ac.nz)

T. Raita, Sodankylä Geophysical Observatory, University of Oulu, F-99600 Sodankylä, Finland. (tero.raita@sgo.fi) 